\title{
7 Complications and Their Avoidance
}

Implantable cortical stimulation therapeutically is subject to the same risks as any typical surgical procedure, such as infection or other wound healing concerns and hematoma formation. Additional complications (such as seizures) may result, however, from cortical mapping necessary to place electrodes, from anchoring the electrodes (migration, hematoma, or CSF leak), tunneling wires (perforation, infection, erosion, significant tissue damage in the neck and or chest), longevity of the device (wire breakage, IPG fault, or lead migration), or in programming the device itself (seizures, sensory effects). Each of these potential pitfalls will be addressed here with advice as to how they may be avoided.

A comprehensive review of over 800 cases in the literature reveals that the extradural approach is not associated with mortality or disabling morbidity, whereas subdural implantation has been associated with significant subdural hemorrhage resulting in postoperative vegetative state (1 patient) and death (1 patient) (Saitoh and Hosomi 2009). Seizures (19 patients) were experienced, but epilepsy was never induced during follow-up, with 1 exception in the 1990's. Aphasia (1 patient) was also observed due to inadvertent overstimulation during the test period (see ahead). Post operatively, infection remains the most commonly reported reason for failure of a device, requiring either a reoperation or removal of the system. The most common significant programming side effect was seizures, occurring in a reported 17 patients. In particular, since 2008, there were 12 seizures and 11 infections.

In this chapter, I will cover basic complication avoidance and provide surgical management tips which may mitigate eventual complications.

\subsection{Complications in Cortical Mapping}

The most likely complication during the mapping phase of the surgical procedure is the generation of seizures. In our experience, seizures have occurred in approximately $25 \%$ of cases while attempting to delineate the M1 region, although all but one of these involved patients who had a history of seizures. Certain precautions may be taken to mitigate their occurrence and the method of mapping out cortical areas itself can significantly lower seizure risk. We have adopted both locating where the N20 somatosensory potential reverses polarity (the location of the CS) and direct cortical stimulation using a ball probe electrode on the surface of the dura and analyzing EMG responses in the face, arm and hand. The N20 potential may be appreciated and localized using either the implant electrode itself or, perhaps more easily adopted for the purpose, a 4 contact strip electrode typically used for intracranial epilepsy 
monitoring. Direct stimulation of the dura can be accomplished using a ball probe electrode with a diameter of $2-4 \mathrm{~mm}$, referenced to an electrode placed on the ipsilateral forehead. It is very helpful to work with a physiologist for these mapping techniques as the surgeon can concentrate on making sure the field stays clear of fluid and blood and can manipulate the electrodes and their location and contact stability to obtain the best signals. The physiology colleague may concentrate on making sure the system is working correctly, troubleshoot and fix technical problems should they arise in unsterile connections, and make continuous changes to amplitude or other parameters while the surgeon focuses more on localizing and moving the electrode to assess the appropriate orientation of the M1 gyrus and CS. In this way, the surgeon may move the electrode strip around after each N20 measurement while the physiologist calls out where the reversal of the potential polarity arises (i.e between which contacts on the strip the reversal occurs, if any). The surgeon may use a sterile marking pen on the dura to denote where this occurred and then move the strip to a different location and orientation for another measurement. Typically 6-8 assessments are made in this way and a reasonable mapping of the M1 region and CS can be found using the SSEP polarity reversal alone. We add direct stimulation mapping as well, because it can provide a second validation to the N20 map, but it also can clarify areas that may have been questionable with the reversal potential technique. The reversal potential is not always easily discerned and may be too broadly localized within the region making it difficult to place the final implanted electrode with assurance. The dipoles of the N2O and the geometry of the CS do not always correlate. Direct stimulation, however, is the most likely part of the technique to cause seizures. We have found that recording from the 4-contact strip electrode placed now over the dura but extending under the edge of the craniotomy opening and out of the way of the field for mapping can serve as a means of assessing cortical epileptiform activity during this stage. It is important not to leave the ball probe on any one area of stimulation too long as well. There is no single limit on this timeframe but more than 2-3 seconds is probably a good rule of thumb. It is better to use a train of 5 stimulus pattern if possible, because this has been shown to have a lower chance of causing seizures. Typical amplitudes to obtain EMG reliably are between 8 and $20 \mathrm{~mA}$, though increasing up to $25 \mathrm{~mA}$ is not unreasonable if no EMG is found (i.e. just to establish that the system is delivering current, there are no technical or noise problems, and that the anesthetic technique is optimal). Consider also that one can stimulate exposed soft tissue such as the temporalis muscle to determine whether the system is delivering current. Mapping the hand region is the most reliable in this setting, having the highest density of representation, though reproducible responses to arm and face regions can usually be obtained with persistence. Finding flank, back, or lower extremities from an extra-dural approach is not reliable. It is useful to plan the craniotomy opening to account for the difference in face location on the M1 surface relative to hand or arm. It is usually significantly more latero-inferior and this can be difficult to reach if the craniotomy opening is not made appropriately, leading 
the surgeon to conclude erroneously that there is a technical problem or they simply cannot find the face region. Figure 7.1 shows representative cranial openings that can avoid these complicating hurdles. Using a functional MRI obtained pre-operatively (e.g. for hand localization) may help in craniotomy planning, or using historically tried-and-true scalp measurements (Fig.7.1) can be just as reliable in our experience. In line with this, the opening itself is best made to allow for the flat alignment and anchoring of the lead to the dura without compromise. Our experience suggests that a large craniotomy ( $>6 \mathrm{~cm}$ in diameter) is not typically necessary, but that up to $5 \mathrm{~cm}$ in diameter, located appropriately with forethought, is very reasonable for moving the electrode around for the N20 and mapping enough of the M1 orientation with the ball probe, as well as for aligning and anchoring the lead adequately (discussed further below). Of course, as outlined elsewhere (chapters 4-5), the electrophysiology part can be skipped and burr holes have proven equally effective; also, one can make a single bur hole and do some mapping with a strip electrode placed through the bur hole in various directions. Finally, should a seizure occur during mapping, it is helpful to have readily available a source of iced saline to irrigate the dura with. This cooling can be useful in terminating the seizure activity (recall that the patient is already under general anesthesia with a TIVA technique - usually Propofol). Avoiding the addition of medication which may compromise mapping is helpful, though if more than one seizure is generated during the mapping process, it is probably best to abort the mapping and decide either to place the electrode using the extant information, or simply to abort the entire procedure. One or two seizures during mapping under anesthesia is not associated with causing any future seizure disorder, within bounds of our accumulated current knowledge.

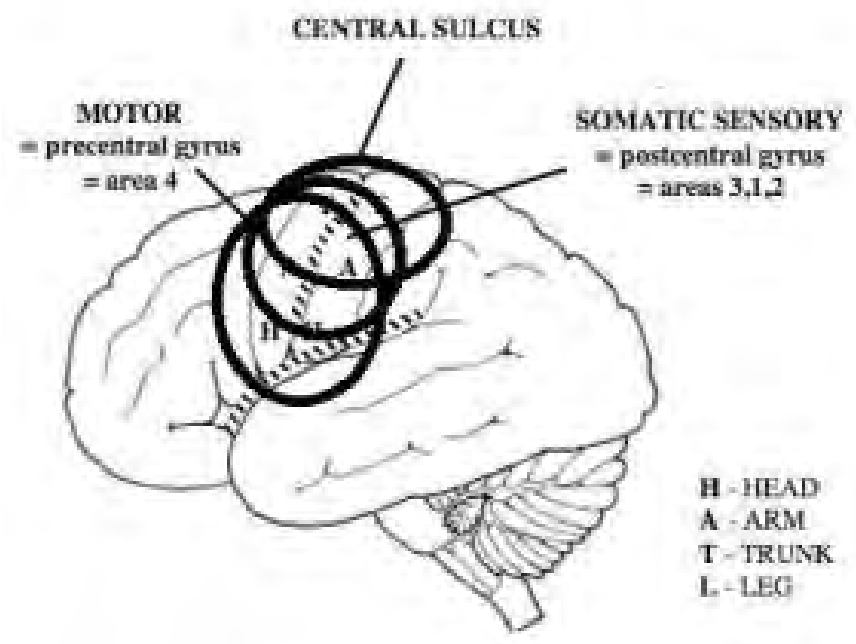

Fig. 7.1: Schema showing examples of craniotomy locations in order to map, for example, arm and flank (more superiorly) versus face (inferior). Pre-planning these adjustments will result in a smaller but adequate craniotomy opening, adequately allowing for manipulation of the electrode during mapping procedures and securing the electrode once final positioning is determined. 


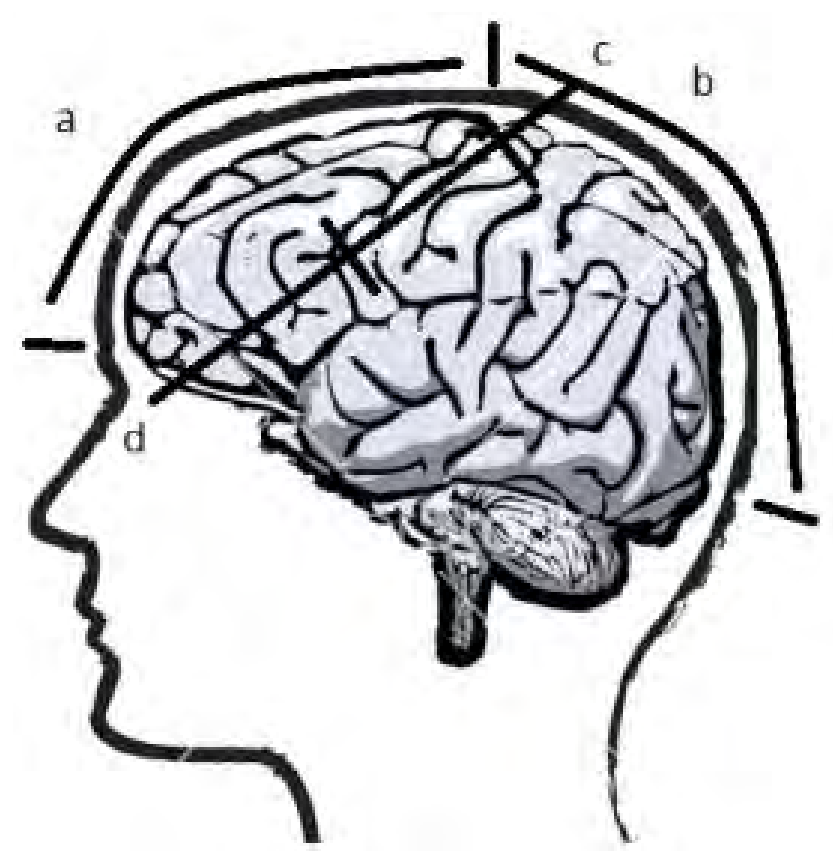

Fig. 7.2: Schema showing basic landmarks for reliably determining the general orientation of the central sulcus. One measures from the nasion to inion, finds the midpoint (vertical line at the top of the head in Figure so that $a=b$ ), and then follows a line extending from approximately two centimeters posterior from this midpoint (ie point c) to just posterior to the lateral canthus (point d). Making use of this information, with or without adjuvant functional imaging or physiology, is helpful and may be the only available means of planning the craniotomy if adjuvant procedures fail, which, of course, can happen.

\subsection{Complications in Snchoring}

As with most neuromodulation devices involving electrodes (Arle and Shils 2011), it is important to minimize the possibility of lead migration by using appropriate anchoring techniques and also perforating the tiny metallic contacts and wires into the electrode. The concepts of 'strain relief' and not kinking the insulation around the wires with sutures are both significant. Figure 7.3 shows typical orientations for our leads secured to the dura, the route of the proximal part of the wire on the dura providing strain relief, and the exit through the appropriately placed bur hole inferiorly and posteriorly, allowing the typical further route of the wire to the IPG to be relatively unimpeded. All leads are surrounded by fibrosis entirely within weeks and the lead (a paddle-type lead) will not ever move once that happens. As with epidural spinal cord stimulation lead placement, this scar formation is sufficient at about 6 weeks post-op. 

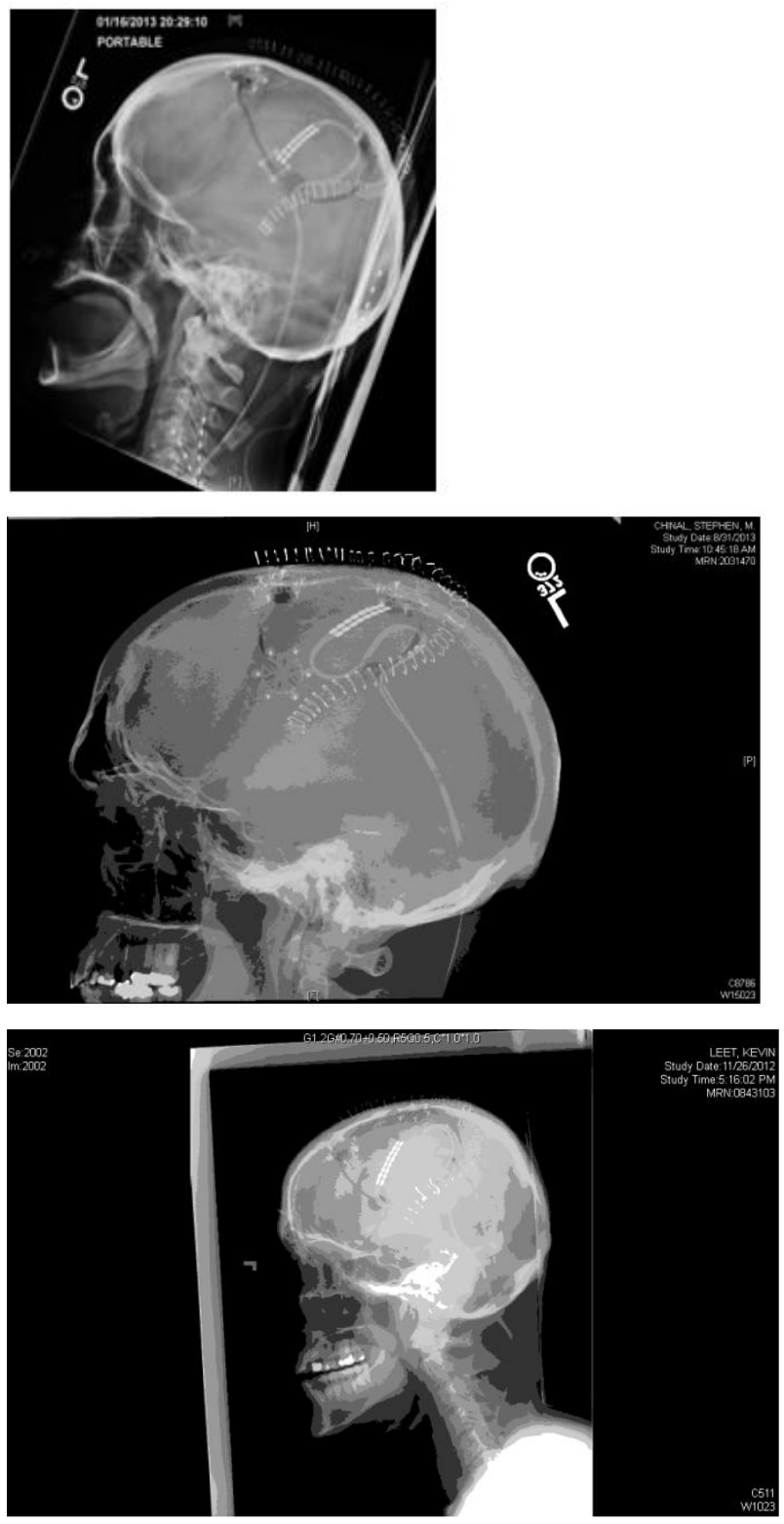

Fig. 7.3: Three representative MCS post-operative lateral skull films that show the size and location of the craniotomy, the lead orientation, the course of the wire as it makes its way to the bur hole and then the exiting of the wire from the bur hole toward the subclavicular region and IPG.

Until then, however, it is important to keep the lead as flat to the dural surface as possible to prevent significant fibrosis or highly non-linear fibrosis occurring between the lead and the dura. A few well-placed 4-0 Neurolon sutures, taking care not to 
damage the wires within the silicone are usually adequate to accomplish this. Damage to the wires within the lead should be carefully avoided when placing these sutures. Some electrodes have mesh within the silicone which makes them more resilient to anchoring; some leads have more room around their edges to accept a stitch. Where there is little room or no mesh, one must be particularly vigilant. Placing a piece of dry gelfoam between the bone flap and the lead during closure is useful as well, both to minimize bleeding, and thus fibrosis, and to hold the lead well against the dura. Migration of these leads early (within 6 weeks of surgery) has been non-existent in our experience if the lead is sutured to the dura and the wire is coiled around the dura before exiting the bur hole and tunneled. A suture or two may be helpful in directing the course of the wire as it coils on the dura before exiting the bur hole. All of these securing sutures should be made in the outer layer of dura only to avoid creating CSF leakage. The use of a larger grid or two paddle leads should be constrained in the same fashion and we have also had no migration or breakage issues in those cases. One or two wires may exit through the same bur hole.

\subsection{Complications in Tunneling and IPG Placement}

Although passing device components (shunt tubing, wires) from the skull region to the subclavicular space or elsewhere has been performed for decades with relative safety and reliability, there are potential pitfalls to be avoided. The main worry is that there will be inadvertent damage to tissues in the posterior or lateral neck, or that the tunneler will pass under the clavicle into the lung or subclavian vessels. Any and all of these complications have no doubt occurred somewhere, but they seem to be very rare. Such complications are unlikely to be reported in the literature. Several tips to keep in mind when tunneling are given below.

- Different tunnelers have different stiffness, sharpness, and ability to be manipulated by the way they are designed to be held. Be aware of these constraints before starting to tunnel. Some tunneler handles may loosen when they need to be secure and help create leverage.

- It is strongly advised to bend the tunneler, perhaps even more than seems appropriate, before tunneling. It is much easier to steer and manipulate the tip of the tunneler when under the skin if it is already bent (generally upwards relative to the direction of passage) than if one has to bend it after it is half or $3 / 4^{\text {th }} \mathrm{s}$ to its target.

- If the tunneler is bent (as suggested) it is then even more important to maintain vigilance in preventing the tip from piercing the skin before reaching the target.

- Keeping the tip bent up is helpful in avoiding other tissue damage and in avoiding allowing the tip to pass inadvertently below the clavicle potentially damaging the lung or subclavian vessels. However, it is also important to keep the tip from 
turning too far right or left as it is being passed - a lack of attention in this regard may bring damage to nearby tissues in the neck.

- Finally, it is not too uncommon for there to be venous bleeding from passage of the tunneler, somewhere along the track. First, determine if it is from the edge of where the tunneler entered or is accessible to being cauterized with the bipolar. If not, then it is imperative that pressure be uniformly applied along the tunnel track, checking periodically to see if bleeding has stopped. Usually this takes at least 5 minutes of direct pressure. Avoiding potentially life-threatening hematoma formation in this manner is obviously very important. In our experience, this bleeding is always venous in origin and while sometimes brisk, always stops with adequate attention to pressure and time.

Most IPG's are going to be placed in the subclavicular region. Several important details should be kept in mind when using this area in order to minimize complications.

- The pocket should be made medial to the deltopectoral groove, approximately two finger-breadths below and parallel to the clavicle, and to a depth at or just anterior to the pectoralis fascia.

- A discussion should always take place pre-operatively, especially with female patients, as to whether placement in the axillary region or under the breast is at all desired. The paraumbilical region can be very convenient in fat persons, but sometimes painful in skinny patients because of frictions with trousers, belts or other clothes. Some young ladies may not accept subclavicular placement of the IPG for esthetic reasons: in these cases, the stimulator can be placed $5 \mathrm{~cm}$ lower and $10 \mathrm{~cm}$ more laterally with a vertical incision above the breast, just on the pectoral muscle (Pirotte et al 2009).

- A dissolving suture (such as a 4-0 monocryl) may be helpful cosmetically in this location but it is advisable to see the incision during healing anyway within 1-2 weeks post-operatively.

\subsection{Complications in Device Hardware}

We have devised or use several ways of assessing device hardware from outside the body (Shils et al 2008), but often the ultimate etiology of a problem can only be determined if the IPG and lead are directly accessed at surgery and tested. This is because radiological evaluation cannot always visualize a wire breakage, especially if it is intermittent with certain positions or movements, impedance testing is unreliable in determining exactly which contacts or any contacts have failed, IPG assessments have limited ability to determine failure modes and recharging failures, and skin recordings, even with palpation and manipulation to determine a short, are not always successful. The implanted pulse generator (IPG) can accidentally turn off due to electromagnetic interference from household devices in close $(<10 \mathrm{~cm})$ proximity, such as electric 
appliances of any kind, but also anti-theft devices and metal detectors or magnets in loudspeakers. The so-called radio test may be useful: IPGs emit a signal at 500-550 $\mathrm{kHz}$ which can be received as a continuous hum on a small battery operated AM radio receiver. Avoiding hardware complications, however, is more difficult. This is because some hardware complications are from intrinsic faults within the IPG and because some problems with hardware (painful IPG site, painful incision area near anchor, broken wires, disconnected wires, displaced lead location, or infection and erosion of wires or the IPG) are more related to patient comorbidities (diabetes, steroid use, thin and poorly perfused skin and soft tissues, and low pain thresholds) than anything else. Certainly, the surgeon can take pains to minimize some of these concerns, but it is likely that some will still occur despite taking every precaution. The old adage that if you don't have any of these complications occur, you probably haven't done enough cases (or you avoid the difficult cases) likely has a ring of truth to it. Below are some suggestions toward minimizing hardware risks for cortical stimulation procedures:

- Always mention to the patient pre-operatively that they may have discomfort from the IPG or that the wire may become tethered in the neck, and in some cases these may need to be revised and in rare cases it is problematic enough that the system may need removal. Managing expectations up front is helpful.

- Have patients generally avoid any significant movement (reaching, twisting, high-level activities) for the first 6 weeks after surgery.

- Avoid making the subclavicular incision too close to the clavicle - this error almost always leads to pain from the IPG pushing against the clavicle.

- Avoid leaving too little soft tissue coverage over the IPG, difficult in thin patients. This tissue shrinks away anyway following surgery and when the coverage is too thin, pain typically follows, or erosion in atypical cases.

- While it is often helpful to make a second incision in the retromastoid region to help with tunneling, this area can more likely become infected or eroded and open. This is because there is little coverage and the blood supply is often compromised. Try to have the wire pass under one side or the other rather than directly under the incision.

- Make sure there is some strain relief coiling under the IPG. Although this will also scar in place, it typically retains some ability to relieve tethering when the head is turned (personal observation under anesthesia).

\subsection{Complications in Programming}

This subtopic may seem unnecessary perhaps, but we have found that it is worth mentioning for a few reasons. First, in the USA, programming of cortical stimulation patients often falls to the company representatives (though we still often do it ourselves), and it is important to work with the same rep or reps often enough with these systems to avoid complications (or simply inadequate programming). Second, 
there is little feedback from patients during programming and little in the way of algorithms to help with programming, making most of the process one of hit-or-miss and guesswork. In general, we try to cover as much of the cortical region as possible and slowly increase the amplitude over a period of months until we see positive results, and then work our way back to minimize battery use while maintaining efficacy. Unfortunately, increasing amplitudes can inadvertently lead to seizures during the programming phase and that becomes the boundary or ceiling for programming. Seizures tend to occur when amplitudes are set above approximately $7 \mathrm{~mA}-$ but our own data shows we have caused seizures when amplitudes have been set (in different patients) at: $3.4 \mathrm{~V}, 14 \mathrm{~mA}, 7 \mathrm{~mA}$, between 4 and $8 \mathrm{~mA}$, and $9 \mathrm{~mA}$. Seizures tend to be focal motor seizures and can be stopped quickly by turning off the stimulator immediately in the clinic. However, they can generalize and they can occur after the patient has left the programming session with the typical unpredictability that seizures have. We recommend the following guidelines in programming to minimize the likelihood of seizure generation.

- Start at 2V or 5-6 mA and leave at that level for the first two weeks.

- Increase amplitude only in $0.1 \mathrm{~mA}$ or volt steps and make no adjustments at less than 4 week intervals.

- Do not exceed amplitudes wherein there is likely driving of the motor system itself (causing speech slurring, contractions, or other strange feelings) - if no benefit occurs at this 'motor threshold' for approximately 6 months, then it is worth informing the patient that they are unlikely going to reap any benefit with the device. However, it is still worth making some changes in PW and frequency anyway to see if there are benefits. Importantly, cycling on/off to preserve battery power may be imperative to keep the patient from having to recharge too often, and switching between several programs may keep the system from 'habituating' to one setting (there seems to be a benefit in some patients to the 'novelty' of a program and the circuitry responds for a short period of time before failing). Also, the patient may not realize they have some benefit until the device is turned off entirely for several days at which point the patient may appreciate that there was benefit. This technique is always worth pursuing when benefit is questionable.

A couple of caveats. Too many of these patients, who do not 'feel' any of the stimulation, are all too aware of when their IPG battery runs down and needs to be replaced without any prompting to be coincidence. Significant others too often notice appreciable improvements in interaction, mood, and activity levels, even when patients themselves may not notice, to dismiss the therapy out of hand. 


\subsection{Other Complications}

Some patients report painful sensations either at the scalp - around the bone flap - or at the supramastoid region - where the connection is located - or around the neurostimulator. Visual inspection, as well as CT, X-rays or MRI usually do not show anything wrong. Pirotte et al (2009) experienced two patients reporting a painful sensation along the subcutaneous trajectory of the extension cable in the neck as if it was stretched like a rope in rotatory movements of the head. Indeed, a fibrous reaction can occur around the extension cable, so that it is best not to tunnel it too superficially in the neck in order for the platysma muscle to cover it completely. It should be borne in mind that normal sensory perception is generally disturbed in neurogenic pain patients, even in places located far from the painful limb or segment. This may cause minor - but chronic and sometimes disabling - complaints centered on the sites of implantation of the electrodes, cables or stimulators.

As classically observed in all surgical disciplines, the risk of infection is increased by the presence of implanted materials. Infection can occur as a primary process, directly related to the electrode implantation and rapidly presents as epidural or subdural (if the dura has been opened - see above) empyema. Infection can also start at the cutaneous exit of the electrode. Finally, infection of the electrode may occur secondarily to incomplete healing of the skin at the level of the scar on the vertex (wound dehiscence). Erosion of the connector through the skin is possible. Placement of the connector in the soft tissues of the neck should be avoided, given the stress related to cervical motion and the connectors' tendency to erode through the skin or break. In practice, the infection rate is not high. Pirotte et al (2009) experienced infections in 3 cases only (1 primary epidural empyema 15 days after surgery, 1 subdural empyema 10 days after surgery and 1 epidural empyema with cortical abscess secondary to incomplete healing of the scar 6 weeks after surgery): all 3 cases required surgical removal of the entire stimulating system, followed by 4 weeks of intravenous antibiotics. In order to avoid primary infection of the electrodes, aseptic measures must be carefully enacted throughout the procedure. Double gloving, avoiding implants manipulations, as well as systematic use of 24-hour-prophylactic intravenous antibiotics are mandatory. If epidural or subdural infection occurs, a cycle of intravenous antibiotics can be attempted, but, in almost all cases, explantation of the whole system is the only effective way to achieve remission. Local cutaneous infection at the exit of the temporary cables behind the ear is benign and rather infrequent, in spite of the cables lying externally for weeks (several centers keep the patients on antibiotics as long as the connection cables are externalized, though). Daily application of an antiseptic (e.g. Betadine) at the local site is usually sufficient to control local inflammation and impede progression of the infection to the epidural space. Secondary infection of the scar due to incomplete healing of the wound at the convexity should not be underestimated. It represents a major source of electrode infection that can lead to forced explantation. Indeed, an incomplete healing of the 
wound can contaminate the subcutaneous space, where the electrode wires can be affected. Pirotte et al (2009) experienced one case of secondary epidural infection of the electrodes complicated by a cortical abscess; another patient developed an epidural empyema secondary to suboptimal wound healing and a cortical abscess just below the electrode. This abscess was visible on CT and MRI and lasted for 6 months before disappearing. A good solution is to draw a large skin incision on the convexity so that the wound would not overlie any bone fixation or any wire or material, the skin incision being far away from the edge of the craniotomy. Finally, skin closure must be technically perfect.

Montes et al (2002) analyzed event-related potentials (ERPs) and behavioral performance during an auditory target-detection task in 11 consecutive patients obtained during MI ECS and 10 minutes after switching off stimulation. While sensory responses remained unaffected by MCS, there was a significant delay of brain potentials reflecting target detection in the older patients (N2 and P3), rapidly reversible after MI ECS discontinuation. No effect was observed in patients younger than 50 years. Individually, the effect was highly variable from no effect to a delay of tens of milliseconds. Cognitive effects of MCS appeared as mild and non-specific, directly related to the stimulation period (i.e. with no post-effect), in a manner reminding of cognitive effects reported during MI rTMS. Thus, MCS may interfere with relatively simple cognitive processes such as those underlying target detection, notably in the elderly and in the presence of preexistent cerebral lesions.

\section{References}

Arle JE, Shils JL. Essential Neuromodulation. San Diego : Academic Press, 2011

Montes C, Mertens P, Convers P, et al. Cognitive effects of precentral cortical stimulation for pain control: an ERP study. Neurophysiol Clin. 2002;32:313-25.

Pirotte BJM, Voordecker P, Levivier M, et al. Principles of surgical implantation and complications avoidance. In: Canavero S (ed) Textbook of therapeutic cortical stimulation. New York: Nova Science, 2009, pp 33-44

Saitoh Y, Hosomi K. From localization to surgical implantation. In: Canavero S (ed) Textbook of therapeutic cortical stimulation. New York: Nova Science, 2009, pp 17-32

Shils JL, Alterman R, and Arle JE. DBS Fault Testing. In: Tarsy D, Okun M, Vitek J, and Starr P (eds) Deep Brain Stimulation in Neurological and Psychiatric Disorders. Humana Press, 2008, pp 473-95 